\title{
RESULTADOS DO TRANSPLANTE HEPÁTICO EM PORTADORES DE HEPATOCARCINOMA
}

\author{
Mônica Beatriz PAROLIN ${ }^{1}$, Júlio Cézar Uili COELHO² ${ }^{2}$, Jorge E. Fouto MATIAS ${ }^{3}$, \\ Vanessa PUCCINELLI ${ }^{4}$, Rossano JARABIZA ${ }^{5}$ e Sérgio O. IOSHII ${ }^{6}$
}

RESUMO - Racional - O hepatocarcinoma é uma das doenças malignas mais comuns em todo o mundo. O transplante hepático é boa opção terapêutica para os pacientes com hepatocarcinoma em fase inicial, alcançando índices de sobrevida semelhantes aos encontrados nos pacientes cirróticos transplantados sem malignidade hepática. Objetivo - Avaliar a evolução de pacientes cirróticos com hepatocarcinoma submetidos a transplante hepático no Serviço de Transplante Hepático do Hospital de Clínicas da Universidade Federal do Paraná, Curitiba, PR. Métodos - Estudo retrospectivo dos pacientes cirróticos com hepatocarcinoma submetidos a transplante ortotópico de fígado no periodo de setembro de 1991 a setembro de 2000. O diagnóstico do tumor foi estabelecido durante os exames pré-transplante em cinco doentes e foi achado incidental no fígado nativo em três. Nos pacientes com diagnóstico pré-operatório de hepatocarcinoma adotou-se como critério de elegibilidade para o transplante, a presença de tumor único de diâmetro $\leq 5 \mathrm{~cm}$ ou até três lesões com diâmetro $\leq 3 \mathrm{~cm}$ cada, ausência de invasão tumoral da veia porta ou de metástases extra-hepática. Foram avaliados como parâmetros principais a sobrevida do paciente e a ocorrência de recidiva tumoral após o transplante. Resultados - $A$ principal causa de doença hepática pré-transplante foi a hepatite por vírus C (50\%). No exame do fígado explantado, a maioria dos pacientes (6/8, 75\%) tinha lesão única; um paciente tinha dois nódulos e em outro detectou-se hepatocarcinoma multifocal incidentalmente no figado nativo. O tamanho do tumor variou de 0,2 a 5,0 cm. Nenhum dos casos apresentou invasão vascular ou linfonodal. Todos os pacientes permaneceram vivos e livres de recurrência tumoral durante o tempo do estudo, sendo a mediana de seguimento de 18,5 meses (variando de 5-29 meses). Conclusão - O transplante hepático é boa opção terapêutica nos pacientes cirróticos com hepatocarcinoma em fase inicial. Com seleção adequada, o transplante hepático oferece excelentes índices de sobrevida livre de recurrência tumoral.

DESCRITORES - Transplante de figado. Hepatocarcinoma. Doença hepática crônica.

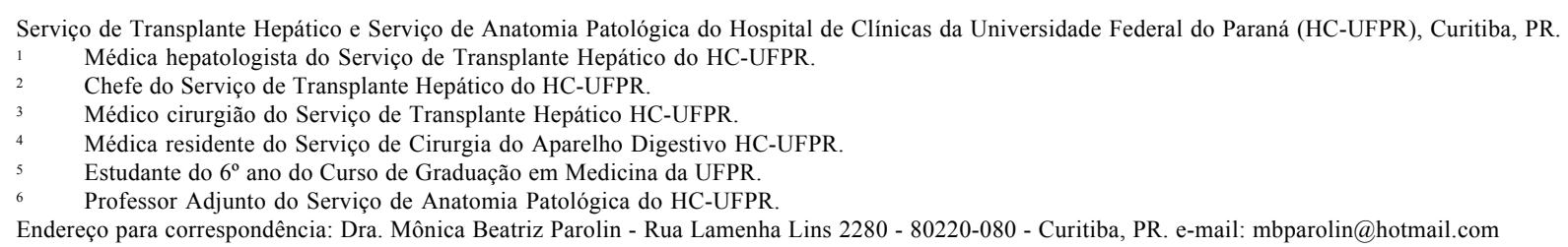




\section{INTRODUÇ̃̃O}

O hepatocarcinoma (HCC) é uma das doenças malignas mais comuns em todo o mundo, com incidência estimada de aproximadamente um milhão de novos casos ao ano. A grande maioria dos HCC se instala em portadores de cirrose hepática, os quais apresentam risco da ordem de $20 \%$ de desenvolver o tumor em $5 \operatorname{anos}^{(3)}$.

A ressecção cirúrgica é a terapia de eleição nas neoplasias primárias do fígado e a única a oferecer a possibilidade de cura. Infelizmente, menos de $30 \%$ dos pacientes com HCC são candidatos ao tratamento cirúrgico no momento do diagnóstico, devido ao tamanho do tumor, a pobre reserva funcional secundária à hepatopatia crônica ou à disseminação extra-hepática. A ressecção cirúrgica do HCC usualmente se restringe aos pacientes com lesões solitárias, com função hepática extremamente bem preservada e sem evidência de hipertensão portal. Nessas condições favoráveis, a sobrevida em 5 anos após a ressecção, pode chegar a $50 \%{ }^{(5,11)}$. Lamentavelmente, a recurrência tumoral após a ressecção cirúrgica é invariavelmente elevada, podendo exceder $50 \%$ em $5 \operatorname{anos}^{(3,12)}$. A recidiva usualmente afeta o parênquima hepático remanescente e pode resultar da disseminação a partir do tumor primário ou ser um carcinoma metacrônico em fígado cirrótico predisposto.

Ao contrário da ressecção cirúrgica que trata apenas o tumor, o transplante hepático $(\mathrm{TH})$ oferece abordagem mais adequada do ponto de vista oncológico pois, além de remover o tumor, substitui o parênquima hepático comprometido por fígado normal, restabelecendo sua função, diminuindo as recidivas em casos selecionados e retirando todo o tecido cirrótico que é pré-maligno. Os primeiros resultados do $\mathrm{TH}$ em casos de HCC foram desapontadores, com índices de recurrência de $48 \%$ a $75 \%$ em 3 anos e sobrevida de $25 \%$ a $31 \%$ em 3 anos, pois incluíam, principalmente, pacientes com doença avançada ${ }^{(8,13,14)}$. A observação de que pacientes cirróticos com tumores pequenos não detectados nos exames pré-transplante (tumores incidentais), apresentavam índices de sobrevida livre de recurrência do tumor indistinguíveis dos cirróticos sem malignidade, reascendeu o entusiasmo sobre o potencial curativo do $\mathrm{TH}$ em relação ao $\mathrm{HCC}^{(12,17)}$. Estudos subseqüentes demonstraram que o estádio do $\mathrm{HCC}$ é fator crucial para a obtenção de bons resultados após o transplante e que quando o TH é realizado em pacientes com tumores solitários com diâmetro $\leq 5 \mathrm{~cm}$ ou até 3 lesões de diâmetro $\leq 3 \mathrm{~cm}$ cada e sem evidência de invasão vascular ou disseminação extra-hepática, a recurrência é desprezível e os índices de sobrevida (75\% em 4 anos) são muito semelhantes aos encontrados nos pacientes sem $\operatorname{HCC}^{(2,10,11,12,15)}$.

O objetivo do presente estudo foi avaliar a evolução dos pacientes cirróticos com HCC submetidos a transplante hepático no Serviço de Transplante Hepático do Hospital de Clínicas da Universidade Federal do Paraná, Curitiba, PR (STH-HC-UFPR), particularmente no que tange às taxas de sobrevida e recurrência do tumor no seguimento pós-transplante.

\section{PACIENTES E MÉTODOS}

Cento e oitenta e três transplantes de fígado (172 transplantes primários e 11 retransplantes) foram realizados no STH-HC-UFPR no período compreendido entre setembro de 1991 a setembro de 2000. Oito pacientes tinham o diagnóstico patológico de HCC no fígado nativo removido durante o transplante. O diagnóstico prétransplante do HCC foi estabelecido pela presença de massa intrahepática consistente com HCC, identificada através de exames de imagem (tomografia computadorizada ou ressonância magnética) e nível de alfa-fetoproteína superior a $40 \mathrm{ng} / \mathrm{mL}$, por lesão hipervascularizada à arteriografia hepática com aspecto característico de $\mathrm{HCC}$, ou por estudo histológico em biopsia dirigida do tumor. Em todos os casos em que o HCC foi detectado na fase pré-transplante, o diagnóstico foi confirmado pelo exame histológico do explante hepático. Tumor incidental foi definido como HCC identificado apenas na avaliação patológica do fígado nativo com exames da fase prétransplante, mostrando nível sérico normal de alfa-fetoproteína $(<20 \mathrm{ng} / \mathrm{mL})$ e ausência de lesão tumoral nos estudos radiológicos. No STH-HC-UFPR, para rastreamento do HCC nos candidatos a $\mathrm{TH}$, são realizadas dosagem do nível sérico de alfa-fetoproteína e ultra-sonografia hepática a cada 6 meses.

Nos pacientes com diagnóstico pré-transplante de HCC, o estádio pré-operatório incluiu a realização de tomografia computadorizada de tórax, abdome e crânio, cintilografia óssea e eco Doppler de veia porta. Como critério de elegibilidade para o $\mathrm{TH}$, considerou-se a presença de tumor único de diâmetro $\leq 5 \mathrm{~cm}$ ou até três lesões com diâmetro $\leq 3 \mathrm{~cm}$ cada, ausência de invasão tumoral da veia porta ou de metástase extra-hepática.

O protocolo adotado pelo STH-HC-UFPR para detecção da recurrência do tumor após o TH consta da realização, a cada 4 meses no primeiro ano, a cada 6 meses no segundo ano e, então anualmente, de alfa-fetoproteína, tomografia computadorizada de tórax, abdome e crânio, e cintilografia óssea

Os prontuários médicos foram revisados para coleta dos seguintes dados: idade na época do transplante, sexo, etiologia da doença hepática, grau de disfunção hepática na fase pré-transplante de acordo com classificação de Child-Pugh, época de diagnóstico do tumor (prétransplante ou tumor incidental), administração de tratamento adjuvante na fase pré-transplante (p. ex. quimioembolização), esquema de imunossupressão adotado, evidência de recidiva do tumor e sobrevida após o TH. Foram também analisadas as características histopatológicas dos tumores identificados nos explantes, tais como número, localização, tamanho (na presença mais de uma lesão considerou-se o diâmetro da maior lesão), presença de invasão vascular macro ou microscópica e presença de infiltração neoplásica em linfonodos.

Os resultados foram expressos como mediana e variação ou percentagens. 


\section{RESULTADOS}

Entre os 172 pacientes submetidos a TH primário foram identificados 8 pacientes com diagnóstico patológico de $\mathrm{HCC}$ no fígado explantado. A amostra estudada compreendeu sete homens $(87,5 \%) \mathrm{e}$ uma mulher (12,5\%) com mediana de idade de 51 anos, variando de 5 a 60 anos. As principais características clínicas dos pacientes portadores de HCC submetidos a TH estão expostas na Tabela 1. A infecção pelo vírus da hepatite $\mathrm{C}$ foi a principal causa de doença hepática crônica no grupo, estando presente em $50 \%$ dos pacientes $(\mathrm{n}=4)$. Três dos oito pacientes apresentavam boa reserva funcional hepática (Child A), sendo o tumor a principal indicação para o $\mathrm{TH}$ (casos 4, 7 e 8, Tabela 1).

Em cinco dos oito casos $(62,5 \%)$ o tumor foi diagnosticado na avaliação pré-transplante. Nos pacientes com HCC detectado no rastreamento pré-transplante, o tamanho da lesão variou de 1,0 a $5,0 \mathrm{~cm}$. Quatro dos cinco pacientes com diagnóstico tumoral prétransplante realizaram quimioembolização arterial do HCC enquanto aguardavam o transplante, com intervalo entre 4 a 18 meses antes do procedimento cirúrgico.

Em três dos oito pacientes com HCC $(37,5 \%)$ o TH foi indicado pela doença hepática avançada, sendo o tumor detectado apenas no exame do explante hepático. Dois dos três apresentavam lesões únicas com tamanho de $0,5 \mathrm{~cm}$ (caso 5) e 4,0 cm (caso 2), respectivamente. Em uma paciente com cirrose biliar primária, identificou-se HCC multifocal no exame do explante (caso 1 , maior nódulo com $0,4 \mathrm{~cm}$ de diâmetro). Na revisão do prontuário médico dos três pacientes com tumor incidental, os exames de ultra-sonografia hepática e alfafetoproteína realizados aproximadamente 6 meses antes da cirurgia, não apresentavam alterações que sugerissem neoplasia maligna hepática.
A análise patológica dos explantes revelou, na maioria dos casos, lesão tumoral única $(6 / 8,75 \%)$, com tamanho variando de 0,2 a 5,0 $\mathrm{cm}$, conforme exposto na Tabela 1 . Em seis dos oito casos, classificouse o HCC como bem diferenciado e em dois como moderadamente diferenciado. Não foram observados linfonodos com metástases ou invasão vascular micro e macroscópica em nenhum dos oito casos.

O esquema de imunossupressão primária adotado foi ciclosporina em microemulsão e prednisona em cinco pacientes e FK-506 e prednisona em três. A mediana de seguimento pós-transplante no grupo estudado foi 18,5 meses, variando de 5 a 29 meses. Até o presente nenhum caso de recidiva foi evidenciado, os pacientes apresentam boa função do enxerto e a taxa de sobrevida é de $100 \%$.

\section{DISCUSSÃO}

Os resultados do presente estudo confirmam que pacientes cirróticos com $\mathrm{HCC}$ em fase inicial podem se beneficiar do $\mathrm{TH}$ se adequadamente selecionados. Na amostra estudada, composta de cinco pacientes com tumores diagnosticados na fase pré-TH e três com tumores incidentais, todos sem evidência de invasão vascular ou disseminação extra-hepática, a sobrevida livre de recurrência tumoral foi de $100 \%$, sendo a mediana de seguimento de 18,5 meses. No presente estudo utilizaram-se como critérios para eligibilidade ao $\mathrm{TH}$ em pacientes cirróticos com HCC, a presença de nódulo solitário de até $5 \mathrm{~cm}$ ou até três nódulos de diâmetro igual ou inferior a $3 \mathrm{~cm}$ cada, baseados na experiência positiva de outros pesquisadores ${ }^{(11)}$. Diversos autores demonstraram que o $\mathrm{TH}$ em pacientes cirróticos com tumores pequenos, sem evidência de invasão vascular ou disseminação extrahepática, associa-se a índices de sobrevida livre de recidiva em 4 anos de até $75 \%$ e com recurrência tumoral aceitável, variando de 3\% a 8\% em $3 \operatorname{anos}^{(2,10,11,12,15)}$. Acredita-se que a seleção rigorosa dos candidatos

TABELA 1 - Características clínicas e tumorais em oito pacientes cirróticos portadores de hepatocarcinoma submetidos a transplante hepático

\begin{tabular}{|c|c|c|c|c|c|c|c|c|c|c|}
\hline \multirow[b]{2}{*}{$\begin{array}{c}\text { Caso } \\
\mathbf{n}^{\circ}\end{array}$} & \multirow[b]{2}{*}{ Sexo } & \multirow[b]{2}{*}{ Idade } & \multirow[b]{2}{*}{$\begin{array}{c}\text { Doença } \\
\text { hepática }\end{array}$} & \multirow[b]{2}{*}{ Child } & \multirow[b]{2}{*}{$\begin{array}{l}\text { Diagnóstico } \\
\text { pré-TH HCC }\end{array}$} & \multirow[b]{2}{*}{$\begin{array}{c}\text { QE } \\
\text { pré-TH }\end{array}$} & \multicolumn{3}{|c|}{ Características do HCC (explante) } & \multirow{2}{*}{$\begin{array}{c}\text { Sobrevida } \\
\text { pós-TH (meses) } \\
\text { sem recidiva }\end{array}$} \\
\hline & & & & & & & $\begin{array}{c}n^{0} \\
\operatorname{les} \tilde{o} e s\end{array}$ & $\begin{array}{l}\operatorname{tamanho} \\
(\mathrm{cm})\end{array}$ & $\begin{array}{l}\text { invasão } \\
\text { vascular }\end{array}$ & \\
\hline 2 & M & 14 & VHB & $\mathrm{C}$ & - & - & 01 & 4,0 & - & 26 \\
\hline 3 & $\mathrm{M}$ & 47 & VHC & $\mathrm{C}$ & + & + & 01 & 4,5 & - & 24 \\
\hline 5 & M & 55 & Criptogênica & B & - & - & 01 & 0,5 & - & 17 \\
\hline 6 & M & 57 & VHC+Álcool & B & + & + & 01 & 5,0 & - & 17 \\
\hline 7 & M & 46 & VHC & A & + & + & 01 & 2,0 & - & 11 \\
\hline 8 & M & 5 & Criptogênica & A & + & - & 01 & 1,5 & - & 5 \\
\hline
\end{tabular}

Abreviaturas: $\mathrm{HCC}=$ hepatocarcinoma; $\mathrm{TH}=$ transplante hepático; $\mathrm{QE}$ - quimioembolização; $\mathrm{CBP}=$ cirrose biliar primária; $\mathrm{VHB}=$ vírus da hepatite $\mathrm{B}$; VHC = vírus da hepatite $\mathrm{C}$.

* diâmetro do maior nódulo tumoral 
a TH e portadores de HCC seja responsável pela excelente sobrevida livre de recidiva observada no presente estudo, apesar desta pequena casuística e do seguimento pós-transplante dessa coorte de pacientes ser limitado, de aproximadamente 2 anos. Embora a recurrência do HCC ocorra na grande maioria das vezes nos primeiros 2 anos após o $\mathrm{TH}$, tal complicação já foi documentada após intervalos maiores ${ }^{(9)}$. Outro fato que contribuiu para os bons resultados obtidos foi a inclusão de três pacientes com carcinoma incidental, grupo que sabidamente apresenta bom prognóstico ${ }^{(1,17)}$.

O TH é o único tratamento que assegura a completa remoção de todos os focos hepáticos de tumor, bem como o tecido sob risco de recurrência tumoral, resultando em índices de sobrevida livre de tumor significativamente superiores aos obtidos pela ressecção tumoral e indistingüíveis dos resultados do TH em cirróticos sem malignidade hepática ${ }^{(3,12,15,17)}$. Entretanto, para que tais resultados sejam alcançados, é fundamental a seleção adequada de pacientes com HCC em fase inicial, de maneira a reduzir a recidiva a níveis aceitáveis, evitando-se o desperdício de órgãos por transplantes fadados ao insucesso. Analisando-se retrospectivamente as primeiras publicações sobre pacientes com HCC submetidos a transplante hepático, constata-se que muitos dos pacientes eram portadores de doença tumoral avançada, já com invasão vascular macroscópica ou disseminação extra-hepática, o que justifica os maus resultados até então obtidos com o $\mathrm{TH}^{(8,12,13,14,15)}$.

A escassez de órgãos é um sério problema a ser enfrentado quando se indica o TH em portadores de HCC. A disparidade entre o número crescente de pacientes em fila para $\mathrm{TH}$ e a escassez de doações tendem a elevar progressivamente o tempo de espera, já superior a 1 ano em muitos centros. Como o tempo necessário para o $\mathrm{HCC}$ dobrar de tamanho estaria em torno de 4 a 6 meses, enquanto esperam em lista, os pacientes correm o risco de haver progressão do tumor para um estádio no qual o TH seja inviável (invasão vascular ou disseminação extra-hepática). Para superar esses obstáculos, diferentes estratégias têm sido utilizadas tais como o emprego de fígados retirados de portadores de doenças metabólicas, tais como a polineuropatia amiloidótica familial (transplante dominó), fígados marginais e, mais recentemente, a possibilidade de realização de transplante inter vivo, utilizando-se o lobo direito do doador ${ }^{(17)}$. Como essas alternativas são acessíveis a poucos candidatos a $\mathrm{TH}$, muitos programas de transplante preconizam tratamento antineoplásico adjuvante durante o período pré-TH através da quimioembolização arterial do $\mathrm{HCC}^{(3,7,17)}$. Na presente série, quatro pacientes com diagnóstico tumoral pré-transplante realizaram quimioembolização arterial do HCC em intervalo variável antes da cirurgia do transplante (4 a 18 meses). A quimioembolização é estratégia atraente devido ao seu efeito antitumoral (isquemia e ação local do quimioterápico), possibilidade de ser realizada periodicamente com boa tolerância e apresentar mínima toxicidade sistêmica ${ }^{(5)}$. O objetivo dessa técnica é induzir a necrose tumoral, reduzindo ou retardando o crescimento da lesão e sua disseminação durante o período de espera em lista.

Recentemente HARNOIS et al.(7) publicaram a experiência da Clinica Mayo na realização periódica de quimioembolização arterial do HCC em pacientes cirróticos aguardando o TH. Vinte e quatro pacientes cirróticos com menos de três lesões tumorais com diâmetro inferior a $5 \mathrm{~cm}$ cada e sem evidência de invasão vascular ou de metástases extra-hepáticas completaram o protocolo de quimioembolizações a cada 2 meses com mitomicina, doxorrubicina e cisplatina. O tempo médio em lista de espera foi de 167 dias. O procedimento foi bem tolerado e nenhum dos pacientes desenvolveu recurrência do tumor após o TH (seguimento médio de 29, 2 meses, variando de 9 a 55 meses). A sobrevida livre de recurrência do tumor em 1 e 2 anos foi de $91 \%$ e $84 \%$, respectivamente. Baseados no protocolo empregado na Clínica Mayo e em outros centros, os autores do presente estudo excluíram a azatioprina do esquema imunossupressor com o objetivo de reduzir o risco de recidiva viral e tumoral, mediante emprego de imunossupressão menos intensa ${ }^{(4,16)}$.

O transplante hepático é hoje a modalidade terapêutica de eleição no STH-HC-UFPR para pacientes cirróticos com HCC inicial, restrito ao fígado e sem evidência de invasão vascular ou disseminação extrahepática. Devido a atual tendência de aumento do tempo de espera em lista para TH, hoje oscilando entre 10 a 12 meses, tem-se adotado a prática de realizar, a cada 2 meses, arteriografia hepática com quimioembolização do HCC, enquanto o paciente aguarda a cirurgia do transplante, baseado nos bons resultados obtidos com essa prática no Programa de Transplante Hepático da Clínica Mayo ${ }^{(7)}$. De acordo com a experiência de HARNOIS et al. ${ }^{(7)}$, tem-se restringido a realização de quimioembolização em pacientes com reserva hepática funcional razoável (Child A e B), pelo risco elevado de grave descompensação de doença hepática em pacientes Child C.

Concluindo, os resultados do presente estudo indicam que o $\mathrm{TH}$ constitui boa opção terapêutica potencialmente curativa em pacientes cirróticos com HCC em fase precoce, associando-se a excelentes índices de sobrevida livre de recurrência tumoral. 
Parolin MB, Coelho JCU, Matias JEF, Puccinelli V, Jarabiza R, Ioshii SO. Results of orthotopic liver transplantation for hepatocellular carcinoma. Arq Gastroenterol 2001;38(4):216-220.

ABSTRACT - Background - Hepatocellular carcinoma is one of the most common malignancies worldwide. Liver transplantation has emerged as a good option for early-stage hepatocellular carcinoma yielding survival rates as good as for recipients without this type of tumor. Objective - To assess the outcome of cirrhotic patients with hepatocellular carcinoma undergoing liver transplantation at the Liver Transplantation Service of the "Hospital de Clinicas", Federal University of Paraná, Curitiba, PR, Brazil. Methods - Retrospective study of cirrhotic patients with hepatocellular carcinoma undergoing orthotopic liver transplantation at the mentioned Institution between September 1991 and September 2000. The diagnosis of hepatocellular carcinoma was established during the pretransplant workup in five patients and the tumor was an incidental finding in the native liver in three. The indication for liver transplantation was restricted to solitary tumor equal to or less than $5 \mathrm{~cm}$ or up to 3 nodules, with each nodule measuring less than $3 \mathrm{~cm}$, and no evidence of vascular invasion or extrahepatic spread. Patient survival and evidence of tumoral recurrence posttransplant were evaluated. Results - The most common cause for pretransplantation liver disease was hepatitis C virus (50\%). On examination of the explanted liver, the majority of patients (6/8, 75\%) had a single lesion; one patient had two nodules and one had a multifocal hepatocellular carcinoma found incidentally in the native liver. Tumor size ranged from 0,2 to 5,0 cm. All cases had neither vascular invasion nor linfonodal envolvement. All patients remained alive and free of tumor recurrence at the time of the study with a mean follow-up of 18,5 months (range, 5-29 months). Conclusion - Liver transplantation is a good therapeutic option for early stage hepatocellular carcinoma arising in cirrhotic patients. With proper selection, liver transplantation can offer excelent survival rates free of tumor recurrence.

HEADINGS - Liver transplantation. Hepatocellular carcinoma. Liver diseases.

\section{REFERÊNCIAS BIBLIOGRÁFICAS}

1. Achkar J-P, Araya V, Baron RL, Marsh JL, Dvorchik I, Rakela J. Undetected hepatocellular carcinoma: clinical features and outcome after liver transplantation. Liver Transplant Surg 1999;4:477-82.

2. Bismuth H, Chiche L, Adam R, Castaing D, Diamond T, Dennison A. Liver resection versus transplantation for hepatocellular carcinoma in cirrhotic patients. Ann Surg 1993;218:145-51.

3. Bruix J. Treatment of hepatocellular carcinoma. Hepatology 1997;25:259-62

4. Burroughs AK. Posttransplantation prevention and treatment of recurrent hepatitis C. Liver Transplant 2000;6 Suppl 2:S35-S40.

5. Fried MW. Treatment of hepatocellular carcinoma: medical options. Liver Transplant Surg 1998;4 Suppl 1:S92-S97.

6. Fuster J, Garcia-Valdecasas JC, Grande L, Tabet J, Bruix J, Anglada T, Taura P, Lacy AM, Gonzalez X, Vilano R, Bru C, Solé M, Visa J. Hepatocellular carcinoma and cirrhosis: results of surgical treatment in European series. Ann Surg 1996;223:297-302.

7. Harnois DM, Steers J, Andrews JC, Rubin JC, Pilot HC, Burgart L, Wiesner RH, Gores GJ. Preoperative hepatic artery chemoembolization followed by orthotopic liver transplantation for hepatocellular carcinoma. Liver Transplant Surg 1999;3:192-9.

8. Iwatsuki S, Gordon RD, Shaw BW Jr, Starzl TE. Role of liver transplantation in cancer therapy. Ann Surg 1985;202:401-7.

9. Klintmaln GB. Liver transplantation for hepatocellular carcinoma - a registry report of the impact of tumor characteristics on outcome. Ann Surg 1998;228:47988 .
10. Marsh JW, Dvorchik I, Subotin M, Balan V, Rakela J, Popechitelev EP, Subbotin N, Casavilla A, Can BI, Eng JJ, Iwatzuki S. The prediction of risk of recurrence and time to recurrence of hepatocellular carcinoma after orthotopic liver transplantation: a pilot study. Hepatology 1997;26:444-50.

11. Mazzaferro V, Regalia E, Doci R, Andreola S, Pulvirenti A, Bozzetti F, Montolto F, Annaturoni M, Morobito A, Gennari L. Liver transplantation for the treatment of small hepatocellular carcinomas in patients with cirrhosis. N Engl J Med 1996;334:693-9.

12. Olthoff KM. Surgical options for hepatocellular carcinoma: resection and transplantation. Liver Transplant Surg 1998;4 Suppl 1:S98-S104.

13. Olthoff KM, Millis M, Rosove MH, Goldstein LI, Raminhg KP, Busuttil RN. Is liver transplantation justified for the treatment of hepatic malignancies? Arch Surg 1990;125:1261-8.

14. Pichlmayr R, Weimann A, Ringe B. Indications for liver transplantation in hepatobiliary malignancy. Hepatology 1994;20:33S-40S.

15. Ranjan D, Johnston TD. Liver transplantation for hepatocellular carcinoma Hepatogastroenterology 1998;45:1369-74.

16. Saxena R, Ye MQ, Emre S, Klion F, Nalesnik MA, Thung SW. De novo hepatocellular carcinoma in a hepatic allograft with recurrent hepatitis $\mathrm{C}$ cirrhosis. Liver Transplant Surg 1999;5:81-2.

17. Suarez Y, França ACV, Llovet JM, Fuster J, Bruix J. The current status of liver transplantation for primary hepatic malignancy. Clin Liver Dis 2000;4:591605 .

Recebido em 6/10/2000. Aprovado em 16/2/2001. 